\title{
The Particle Flow Algorithm in the Phase II Upgrade of the CMS Level-1 Trigger
}

\author{
Dylan Rankin $^{a, *}$ on behalf of the CMS Collaboration \\ ${ }^{a}$ Massachusetts Institute of Technology, \\ 77 Massachusetts Avenue, Cambridge, MA, 02139, USA
}

E-mail: drankin@mit.edu

\begin{abstract}
The CMS experiment has greatly benefited from the utilization of the particle-flow (PF) algorithm for the offline reconstruction of data. The Phase II upgrade of the CMS detector for the High Luminosity upgrade of the LHC (HL-LHC) includes the introduction of both tracking and the new high-granularity calorimeters in the Level-1 trigger. The inclusion of tracking in particular offers the possibility of developing a simplified PF algorithm in the Level-1 trigger, as well as the possibility for the use of advanced pileup (PU) mitigation techniques such as Pileup Per Particle Identification (PUPPI). We present the logic of these algorithms, along with their inputs and possible implementation using prototype hardware. We show that these implementations are capable of operating within the budgeted latency requirements of the Level-1 trigger environment. The expected performance and physics implications of such algorithms are shown using Monte Carlo samples with 200 pileup, simulating the harsh conditions of the HL-LHC. We discuss the importance of these advanced techniques in preserving the physics performance in the highintensity environment.
\end{abstract}

40th International Conference on High Energy physics - ICHEP2020

July 28 - August 6, 2020

Prague, Czech Republic (virtual meeting)

\footnotetext{
${ }^{*}$ Speaker
} 


\section{Introduction}

The High Luminosity upgrade of the Large Hadron Collider (HL-HLC) will bring many new challenges for the CMS experiment. In order to meet these challenges, every component of the detector will be upgraded in some way to handle the new conditions of high pileup and instantaneous luminosity. As part of these upgrades, the Level-1 trigger will receive information from the tracking detector [1,2]. This represents a major expansion in the scope and capabilities of the Level-1 trigger. To take advantage of this new information CMS will implement a simplified version of the particleflow (PF) algorithm used offline for event reconstruction [3]. Along with PF, the Level-1 trigger will also run an algorithm called Pileup Per Particle Identification (PUPPI) [4]. PUPPI will enable CMS to maintain high trigger efficiencies even in the extreme pileup environment of the HL-HLC.

\section{Design}

The layout of the Level-1 trigger upgrade is shown in Fig. 1a. Both PF and PUPPI will be run in a group of processing boards called the Correlator Trigger, which takes inputs from the tracker, calorimeters, and muon systems. Additionally, inputs are also received from the Global Track Trigger, which is responsible for computing the location of the primary vertex in each event.

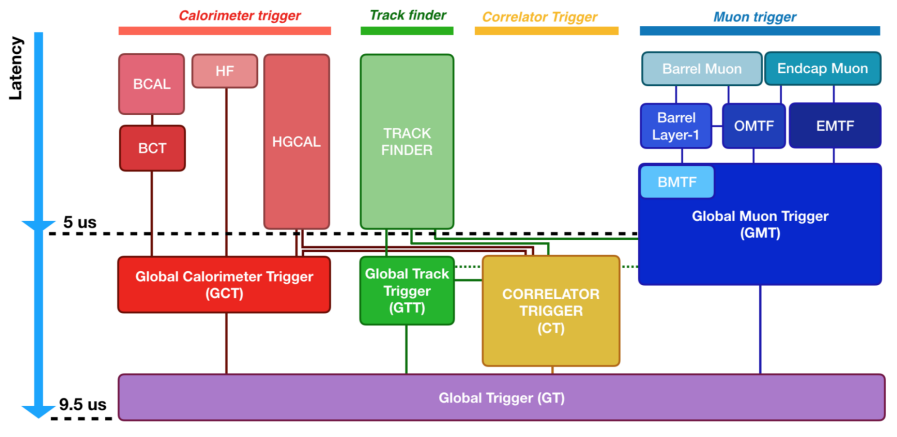

(a) Diagram of the layout of the upgraded Level-1 trigger. The time elapsed from the collision of interest is represented approximately by the distance from the top of the figure.

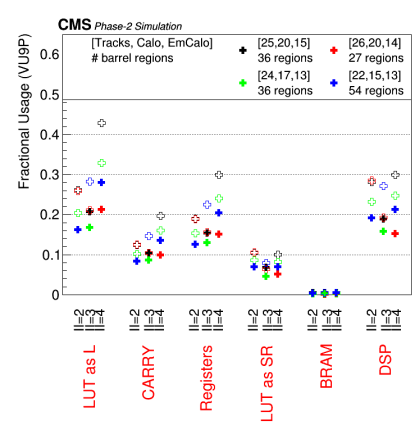

(b) Summary of the FPGA resources required to implement PF and PUPPI for various possible region sizes and initiation intervals.

The Level-1 PF algorithm works by associating information from each subdetector, providing a complete description of the event in terms of particles rather than detector signatures. Standalone muons are associated with tracks to create PF muons, tracks are associated with electromagnetic calorimeter clusters to create PF electrons and photons, and tracks are associated with hadronic and electromagnetic calorimeter clusters to create charged and neutral hadrons. The Level-1 PUPPI algorithm takes as input the PF particles and the primary vertex location along the $z$-axis. Charged particles that originate from close to the primary vertex are then used to compute the PUPPI weight and adjust the assigned transverse momentum of all neutral particles. Since it is not feasible to run PF and PUPPI on the entire detector at once, it is instead segmented into smaller regions, roughly of size $0.5 \eta \times 0.7 \phi$ (depending on the location of the region). These region sizes were chosen to optimally use the FPGA resources, balancing the size of the input region with the size of the PF and PUPPI algorithm blocks needed to process the region and the number of regions needed to 
cover the full detector. The options that were considered for region size and initiation interval are shown in Fig. 1b. The piece of firmware responsible for assigning the inputs from each subsystem to their appropriate regions is called the regionizer. Software emulators for both the regionizer and the PF and PUPPI firmware have been developed and fully validated on simulated $t \bar{t}$ events with 200 additional proton-proton interactions per bunch crossing. All the firmware components and algorithms have also been implemented and tested together using prototype boards with the targeted Xilinx Virtex Ultrascale 9+ FPGA. The utilized percentage of the available FPGA resources of each type is less than $50 \%$ in order to allow for future developments.

\section{Performance}

Across a wide range of physics topologies and signatures, we find that PF and PUPPI are able to greatly improve the performance of the Level-1 trigger with respect to track- or calorimeter-only objects. For jets we find that PF is capable of improving the jet resolution by about $10 \%$ (absolute) at low $p_{T}$ in most regions of the detector. For $H_{T}$ (defined as the scalar sum of the $p_{T}$ of all jets) and missing transverse energy (MET) triggers, PUPPI produces a sharper trigger turn-on and reaches 95\% efficiency at an offline threshold roughly $200 \mathrm{GeV}$ lower than either a track- or calorimeteronly trigger for a similar trigger rate. We also find that a hadronic tau lepton trigger using a neural network based on PUPPI particles is able to lower the turn-on for hadronic tau leptons by more than $40 \mathrm{GeV}$. A trigger that combines track and calorimeter information but does not use PF is able to produce a similar turn-on, but reaches a plateau efficiency approximately $10 \%$ lower than the PUPPI-based trigger for a similar rate. A summary of these results is shown in Fig. 2.
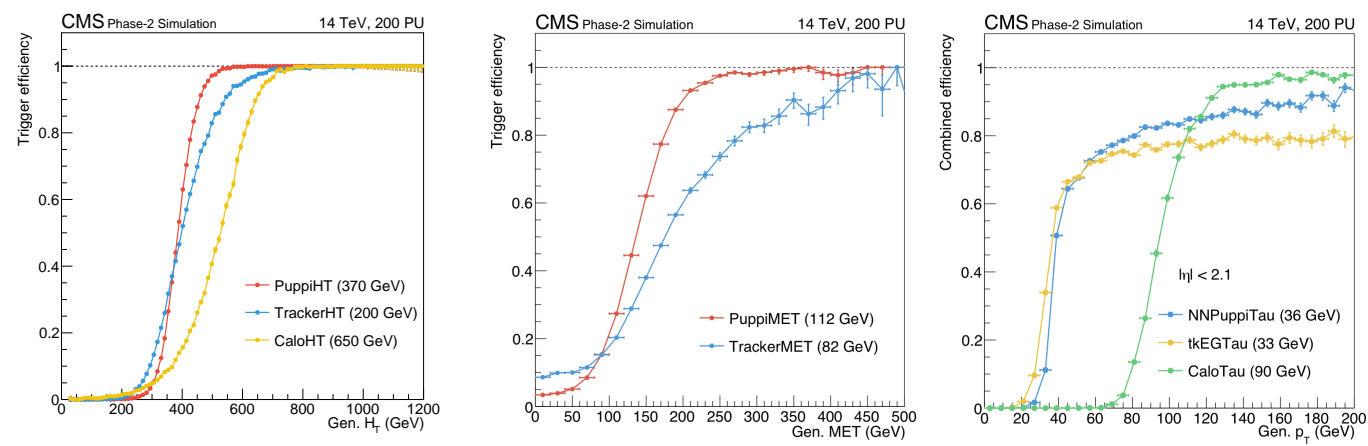

Figure 2: Examples of the improved performance possible when using PF and PUPPI objects as opposed to track- or calorimeter-only objects for $H_{T}$ (left), MET (center), and hadronic tau (right) triggers.

\section{References}

[1] The Phase-2 Upgrade of the CMS Tracker. Technical Report CERN-LHCC-2017-009. CMS-TDR-014, CERN, Geneva, Jun 2017. URL https://cds. cern. ch/record/2272264.

[2] The Phase-2 Upgrade of the CMS Level-1 Trigger. Technical Report CERN-LHCC-2020-004. CMS-TDR-021, CERN, Geneva, Apr 2020. URL http://cds. cern. ch/record/2714892.

[3] A.M. Sirunyan et al. Particle-flow reconstruction and global event description with the CMS detector. JINST, 12(10):P10003, 2017. doi: $10.1088 / 1748-0221 / 12 / 10 / \mathrm{P} 10003$.

[4] Daniele Bertolini, Philip Harris, Matthew Low, and Nhan Tran. Pileup Per Particle Identification. JHEP, 10:059, 2014. doi: 10.1007/JHEP10(2014)059. 